\title{
Optimal Distributed Consensus Algorithm for Fair V2G Power Dispatch in a Microgrid
}

\author{
Mingming Liu \\ Hamilton Institute \\ National University of Ireland Maynooth \\ Maynooth, Ireland \\ Email: mliu@eeng.nuim.ie
}

\author{
Emanuele Crisostomi \\ University of Pisa \\ Pisa, Italy
}

\author{
Yingqi Gu \\ School of Engineering \\ University of Edinburgh \\ Edinburgh, UK
}

\author{
Robert Shorten \\ IBM Research \\ Dublin, Ireland
}

\begin{abstract}
Among the many motivations to encourage the use of Electric Vehicles (EVs) there is the attractive possibility to implement Vehicle-to-Grid (V2G) functionalities. They are attractive both for EV owners, who can sell their own energy to the grid when they do not need to travel, and also for the power grid, as the stored energy can be used to back-up the fluctuating energy produced from renewable sources or to improve the grid stability at critical times. In this paper we illustrate a distributed algorithm that solves the V2G problem in a fair manner, trying to achieve an optimal trade-off between power generation costs and inconvenience to the vehicle owner. Results are shown and discussed for a case study simulated in the OpenDSS power system environment. Keywords: EV, V2G, Decentralised optimisation, Microgrid
\end{abstract}

\section{INTRODUCTION}

One of the most attracting functionalities of Electric Vehicles (EVs) is the possibility to use them as a large virtual battery to support the power grid when needed. In particular, energy can be stored when the grid produces excess energy, and can be delivered back to the grid in times of need [1]-[4]. The attractiveness of having EVs providing energy to the grid, usually denoted as Vehicle-to-Grid (V2G), stems from the fact that EV owners see in this process the possibility of earning money by selling the $\mathrm{V} 2 \mathrm{G}$ service to the grid when the EV is not needed. Also, this service can be provided without inconvenience for the owners, as they simply have to maintain the EV constantly plugged-in, even when it is fully charged. At the same time, there is a high interest in V2G functionalities from the perspective of the smart grid as well. In fact, in a forthcoming scenario when a large share of energy will be provided from renewable sources, it will be possible to mitigate the fluctuations of the renewable energy by storing it when it is in excess, and by recovering it from the EVs when the renewable energy is not enough to match the power load. Note that nowadays most of the support to renewable energy is performed by switching on conventional power plants that are more expensive than solar/wind plants (as there are some fuel and carbon costs to be paid), and also less environmentally friendly in terms of produced $\mathrm{CO}_{2}, \mathrm{NO}_{\mathrm{x}}$ or other pollutants' emissions. In this context, note that some countries are already characterised by a high penetration level of energy produced from renewables, see for instance the case of Denmark, where wind plants alone provided more than $30 \%$ of the electricity production in 2012, and are planned to supply $50 \%$ of the overall demand by the year 2020 [5]. Also, Denmark is currently trying to become a system based only on renewable energy by 2050 [6].

Recent works on $\mathrm{V} 2 \mathrm{G}$ practises can be found in references [7]-[9]. A recent paper on the same topic [10] overviews the optimality criteria that should be considered when planning V2G functionalities. In particular, it shows that V2G functionalities should be planned in a wise fashion, as taking electricity from vehicles in an indiscriminate fashion, could sometimes give rise to an environmental cost that might even exceed the environmental gain of using power generated from renewables (e.g., if the consequence of V2G services is that the EV owner has to take a dirty vehicle because his/her EV does not have enough energy for the next trip anymore).

In this paper we model the $\mathrm{V} 2 \mathrm{G}$ problem as an optimisation problem that aims at finding the optimal trade-off between the economic convenience of using energy from renewables instead of energy from other more expensive power plants, and the inconvenience caused to the owner in terms of residual energy remaining in the EV. Also, we are interested in computing a "fair" solution, where fairness is related to the fact that the same energy should be taken from all EVs participating to the $\mathrm{V} 2 \mathrm{G}$ programme (i.e., some EVs' owners might decide not to participate to such a programme to preserve the level of energy in their EVs for future usage). Note that the difficulty of such an optimisation problem is that the utility functions associated with each EV are strictly personal, as the inconvenience for the owner depends on personal factors. Also, we are interested in computing the optimal solution in a distributed fashion, to avoid increasing computation loading in the central server and improving flexibility and robustness from a system perspective.

This paper is organised as follows: the next section describes the mathematical formulation of the $\mathrm{V} 2 \mathrm{G}$ problem, giving the details of the utility functions, of the battery model, and analysing the proposed distributed algorithm. Section III simulates the proposed algorithms in a microgrid scenario, using the popular power system simulation software 
OpenDSS [11]. Section IV discusses the results obtained in the simulation environment. Finally in Section $\mathrm{V}$ we summarise our findings and outline future lines of research.

\section{Model AND Algorithm}

\section{A. Model Set-up}

In the smart grid framework, a microgrid can be regarded as an efficient and environmentally friendly energy subsystem consisting of Distributed Generators (DGs) and loads capable of operating in parallel with, or independently from, the main power grid [12], [13]. In our context, we consider a microgrid where a number of EVs are plugged-in in a large parking lot, e.g., a parking lot in a city airport. Some of such EVs decide to participate to a $\mathrm{V} 2 \mathrm{G}$ programme: during their parking period, they can be discharged/recharged within some given limits in order to improve the economic operation of the grid. The EVs participate to such a programme to get some economic revenues. The discharge should thus occur in a fair way to avoid having some EVs getting more money than others.

These EVs are coordinated by an EV aggregator in the microgrid. In this scenario, the optimal power discharge rate is computed by taking into account both some economical aspects and some Quality of Service (QoS) of customers. We assume that the grid will charge the EV batteries with excess energy produced from renewable sources in the microgrid. This implies that in the future, when needed, the grid will consider the possibility of taking energy from EVs back to the grid if the load exceeds the energy currently produced from renewables (e.g., peak-time hours). Without EVs connected, the grid would have to use the power generated from conventional sources, thus incurring in high generation costs (e.g., fuel and consumption costs) and in increased harmful pollution (e.g., $\mathrm{CO}$ and $\mathrm{NO}_{\mathrm{x}}$ ). Thus, the economical convenience of using EVs relies in recovering energy from the EVs rather than producing new energy from conventional plants. The QoS aspect of the utility functions takes into account the concerns of the EV owners, as not too much energy should be taken from their EVs or they will have a reduced mobility range.

We mathematically formulate the previous problem as follows. Let $N$ denote the maximum number of EVs participating into the scheme. Define the set $I:=\{1,2, \ldots, N\}$ for indexing all EVs and the set $\phi(t)$ for indexing the EVs available at time $t$, i.e., the EVs with enough SOC for participating to discharge cycles in the scheme. Let $|\phi(t)|$ denote the number of elements in the set $\phi(t)$. Let $c_{i}(t)$ denote the discharge rate of the $i^{\prime}$ th $\mathrm{EV}$ in set $I$. Further, let $c_{\max }^{i}$ represent the maximum discharge rate that can be injected to the grid. Both values are assumed to be positive in the V2G mode. The corresponding discharge rate vector for all EVs at time $t$ is defined as $\mathbf{C}(t)^{\mathrm{T}}:=\left[c_{1}(t), c_{2}(t), \ldots, c_{N}(t)\right]$. In our study, $\mathrm{V} 2 \mathrm{G}$ power dispatch to the grid is discretised into $M$ time slots (indexed as $1,2, \ldots, M$ ) each of length $\Delta \mathrm{T}$. Each EV is scheduled connecting to the grid within certain time slots, which is represented by the range $\left[a_{i}, b_{i}\right]$. Due to technical diversity of batteries and chargers, each EV may have a different battery capacity $(\mathrm{kWh})$ and a different energy transferring efficiency. The parameters for both factors are denoted as $B_{i}$ and $\eta_{i}$ respectively. Furthermore, to protect the EVs from over-discharging, the minimum SOC for $i^{\prime}$ th $\mathrm{EV}$ is defined as $S O C_{\mathrm{min}}^{i}$. This parameter defines the drop out criteria for the EV. Thus, if $S O C_{i}(t) \leq S O C_{\min }^{i}$, then $c_{i}(t)=0$. To this end, given the initial $\mathrm{V} 2 \mathrm{G}$ regulation time as $t_{0}$, the SOC for the $i^{\prime}$ th EV at time $t$ is given by:

$$
S O C_{i}(t)=S O C_{i}\left(t_{0}\right)-\sum_{j=t_{0}}^{t-1} \frac{c_{i}(j) \cdot \Delta \mathrm{T}}{B_{i}}, \forall i \in I
$$

\section{B. Utility Functions}

In addition to the basic model parameters, each EV $i$ is also associated with a utility function $f^{(i)}: \mathbb{R} \mapsto \mathbb{R}$, which models both economical and QoS term of the energy delivered from the $i^{\prime}$ th EV. In our context, this function will be modelled as a convex function arising from combining the two terms. In addition, we will denote the first derivative of the utility function $f^{(i)}$ as $f^{\prime(i)}$.

Now we model the utility functions in detail. As already mentioned, each function is composed of two different terms that take into account the economic and the QoS aspects respectively. A schematic diagram is illustrated in Fig.1 demonstrating the basic function relation of both terms related to the $\mathrm{EV}$ discharge rate.

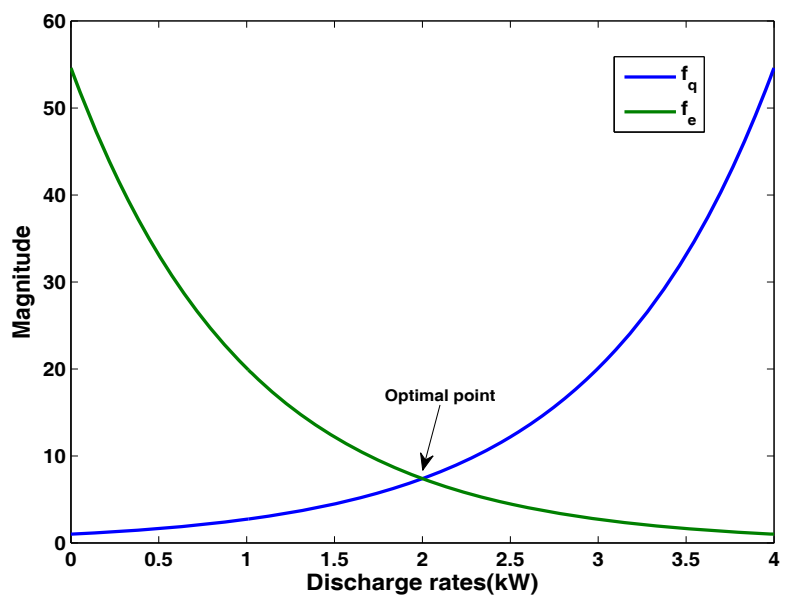

Fig. 1. Schematic diagram of the utility function

Note that in Fig.1 the notation $f_{e}$ and $f_{q}$ refer to the economic term and the inconvenience term of discharge rate (i.e., $c_{i}(t)$ ) respectively. The maximum discharge rate is assumed to be $4 \mathrm{~kW}$ corresponding to the parameter $c_{\max }^{i}$ in our model. According to this diagram, the economic term $f_{e}$ implies that the less is the energy delivered from EVs, the greater is the money required by the grid for purchasing more expensive 
power generation, e.g., conventional power plants. In fact, power generation costs in the literature are typically modeled as a quadratic function of the energy produced in a unit of time [14], [15]. On the other hand, the inconvenience term $f_{q}$ indicates that the more energy is delivered from EVs, the higher is the inconvenience to EV owners. With this idea, we approximately model the overall utility function taking account of both terms as a quadratic function. Considering the difference of the inconvenience term of the users, we perturbed each function with a factor according to the SOC of each EV. To this end, the overall expression of the utility function of the $i^{\prime}$ th EV is modelled as follows:

$$
\begin{array}{r}
f^{(i)}\left(c_{i}(t)\right)=f_{q}^{(i)}\left(c_{i}(t)\right)+f_{e}^{(i)}\left(c_{i}(t)\right)=\alpha_{i} \cdot c_{i}(t)^{2}+\beta_{i} \cdot c_{i}(t) \\
+\left[1-\left(S O C_{i}\left(t_{0}\right)-S O C_{\min }^{i}\right)\right] \cdot c_{i}(t)+\gamma_{i}, \forall i \in \phi(t) .
\end{array}
$$

Comment: In the equation (2), parameters $\alpha_{i}, \beta_{i}$ and $\gamma_{i}$ refer to the pricing function adopted by the grid to give revenues to the EVs participating to the programme. Each perturbed function $f^{(i)}$ is associated with an increasing affine function with positive parameter $\left[1-\left(S O C_{i}\left(t_{0}\right)-S O C_{\min }^{i}\right)\right]$. Thus, the closer is the EV to its safety level of energy $S O C_{\min }^{i}$ required to come back home, the greater is the inconvenience to the owner. In practice, the EV owners can indicate a bigger $S O C_{\min }^{i}$ than the one truly needed, if they are particularly anxious to have enough energy for their next journey. In addition, they can always set the parameter $S O C_{\min }^{i}$ to 1 to be automatically excluded from the $\mathrm{V} 2 \mathrm{G}$ programme.

With the utility functions in place, the overall optimisation problem can be defined as follows:

$$
\begin{gathered}
\underset{\mathbf{C}(t)}{\operatorname{minimise}} \sum_{i \in \phi(t)} f^{(i)}\left(c_{i}(t)\right) \\
\text { subject to: }\left\{\begin{array}{c}
c_{i}(t)=c_{j}(t), \forall i \neq j \in \phi(t) \\
0 \leq c_{i}(t) \leq c_{\max }^{i} \\
S O C_{\min }^{i} \leq S O C_{i}(t) \leq 1
\end{array}\right.
\end{gathered}
$$

\section{Optimal Solution}

In this section we will introduce a theorem that will be helpful for solving problem (3). The detailed proof of the theorem has been given in [18]. Here we briefly recall the mechanism of the theorem for further discussion of the algorithm given in Section II-D.

To begin with, we introduce some notation for the optimisation problem to be considered in (5). Let $n$ denote the total number of agents to be optimised. Let $\underline{n}$ denote the set $\{1,2, \ldots, n\}$. Let $x^{(i)}$ denote the $i^{\prime}$ th entry of vector $x \in \mathbb{R}^{n}$. Each agent $i$ is associated with a function $f^{(i)}: \mathbb{R} \mapsto \mathbb{R}$. Let us assume that each function $f^{(i)}$ is also a strictly convex, continuous and second order differentiable function. Also its first derivative function $f^{\prime(i)}$ has strictly positive and bounded growth rate for $\forall i \in \underline{n}$, that is for all $a \neq b$

$$
0<d_{\min }^{(i)} \leq \frac{f^{\prime(i)}(a)-f^{\prime(i)}(b)}{a-b} \leq d_{\max }^{(i)}, \forall i \in \underline{n}
$$

for suitable constants $d_{\min }^{(i)}, d_{\max }^{(i)}$. The optimisation problem of our interest is now given as follows:

$$
\begin{array}{cc}
\underset{x \in \mathbb{R}^{n}}{\operatorname{mininise}} & \sum_{i=1}^{n} f^{(i)}\left(x^{i}\right) \\
\text { subject to: } & x^{i}=x^{j}, \forall i \neq j \in \underline{n}
\end{array}
$$

Now, we introduce some notation for the Theorem. Let 1 denote the vector with all entries equal to 1 of appropriate length. Define $G$ as the summation of the derivative functions, that is:

$$
G(x)=\sum_{i=1}^{n} f^{\prime(i)}\left(x^{i}\right), \quad \forall x \in \mathbb{R}^{n} .
$$

Define a finite set $\mathcal{P}:=\left\{P^{(1)}, P^{(2)}, \ldots, P^{(m)}\right\}$ of primitive, row-stochastic matrices with strictly positive main diagonal entries.

Now we consider the nonlinear dynamical system:

$$
x_{k+1}=P_{k} x_{k}+\mu \cdot\left(0-G\left(x_{k}\right)\right) \mathbf{1}
$$

Note that, we will denote $x_{k}$ as the solution defined by (7) starting from an arbitrary initial point $x_{0}$.

Theorem 1: Let $\left\{P_{k}\right\} \subset \mathbb{R}^{n \times n}$ be a sequence in $\mathcal{P}$. Then the elements of $x_{k}$ defined by (7) will approach each other for all initial conditions, that is,

$$
\lim _{k \rightarrow \infty} x_{k}^{p}-x_{k}^{q}=0, \forall p, q \in \underline{n} .
$$

Further, for all $\mu$ satisfying

$$
0<\mu<2\left(\sum_{i=1}^{n} \frac{d_{\max }^{(i)}}{d_{\min }^{(i)}}\right)^{-1}
$$

then $\lim _{k \rightarrow \infty} x_{k}=x^{*} \mathbf{1}$, where $x^{*} \mathbf{1}$ is the optimal point of problem (5), that is,

$$
\sum_{i=1}^{n} f^{(i)}\left(x^{*}\right) \leq \sum_{i=1}^{n} f^{(i)}(x)
$$

for all $x \in \mathbb{R}$ and the equality holds if and only if $x=x^{*}$.

\section{Optimal Distributed Consensus Algorithm}

We now describe an optimal distributed consensus (ODC) algorithm that consistently with the Theorem 1 is applied into the practical scenario of interest. Such an algorithm follows from the principles obtained from Theorem 1 and takes into account the practical grid constraints. In the algorithm, $N_{t}^{i}$ represents the set of neighbours of the agent $i$ at time $t$, satisfying $N_{t}^{i} \subset \phi(t)$ and $i \in N_{t}^{i}$, which can send its discharge rate signal to the $i^{\prime}$ th EV. In this work, we assume that each EV can communicate to its neighbours at each time step. Although the neighbours of each EV might change in time, the resulting communication network of all EVs is required to be connected all the time (i.e. there should exist a path from each EV to every other EV in the network). The parameter $\mu$ is chosen in the range discussed in Section II-C, while a detailed discussion on the choice of $\psi$ can be found in [17] and [18]. 


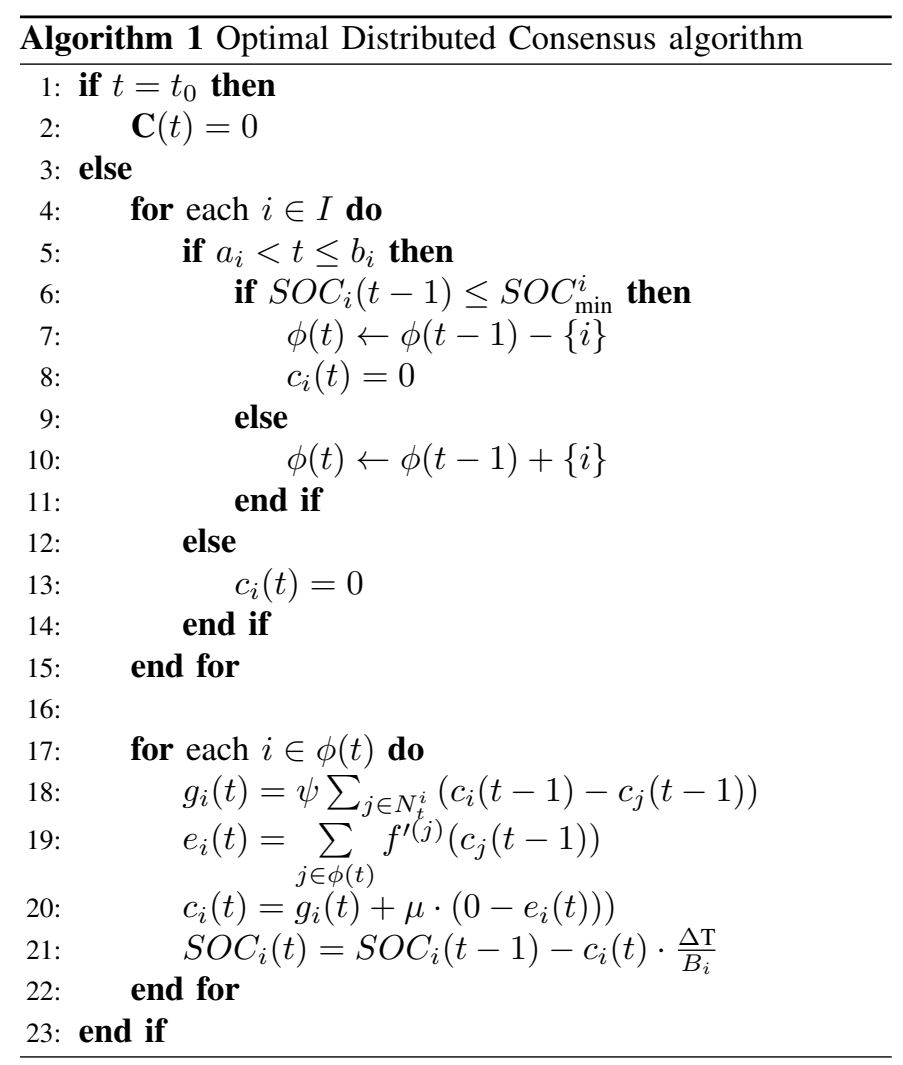

\section{Microgrid Simulation}

To evaluate the performance of the proposed ODC algorithm in a realistic microgrid scenario, a specific microgrid model was constructed through a dedicated power system simulation software OpenDSS. A schematic diagram is illustrated in Fig.2 to demonstrate the structure of the microgrid.

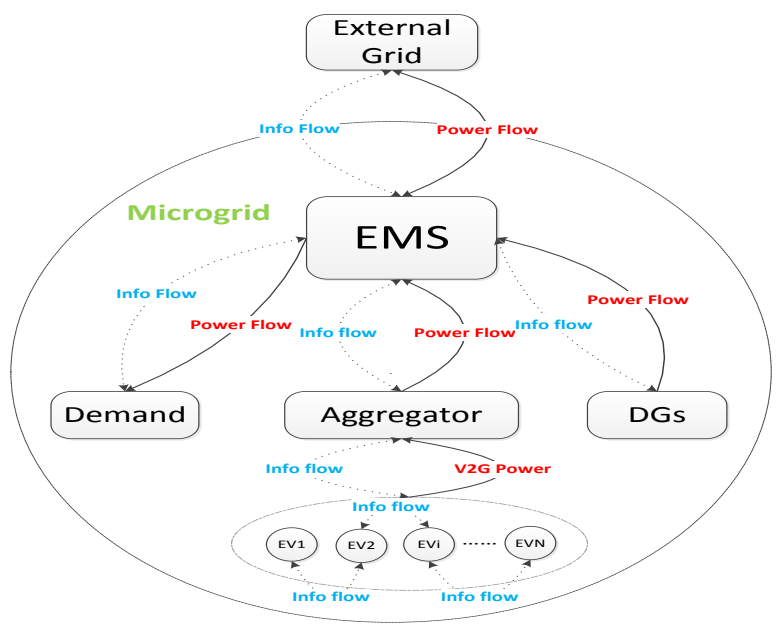

Fig. 2. Schematic diagram of the microgrid

The power topology diagram of this microgrid is shown in Fig.3. In the simulation, we assumed that several DGs were installed in the Medium-Voltage (MV) network with voltage level equal to $10 \mathrm{kV}$. This network included three



Fig. 3. Power topology of the microgrid network

wind plants, two Photovoltaic (PV) plants and one Combined Heat and Power (CHP) plant. These DGs associated with the V2G power inverted from EV batteries were connected through three phase $400 \mathrm{~V} / 10 \mathrm{kV}$ set-up transformers to the MV network. Those DGs were modelled as constant P-Q generators with the same power factor (i.e., 1.0) to generate pure active power for the loads. In practice, we consider a microgrid similar to the one of [16], with a smaller scale to fit into the case of interest here. Due to the space limit, a microgrid topology schematic diagram will be demonstrated in the final version of the paper. The capacity of the wind plants, the PV plants and the CHP were chosen as $600 \mathrm{~kW}$, $184 \mathrm{~kW}$ and $800 \mathrm{~kW}$. The maximum wind power output for each wind DG was randomly chosen from the real wind turbine data according to [20]. In addition, the maximum solar power generation profile of each PV was computed according to a quadratic function with non-zero values from $6 \mathrm{am}$ to $6 \mathrm{pm}$, randomly perturbed to simulate cloud disturbances, as in [21]. We assumed that total demand in the microgrid was composed of 20 basic loads connected randomly in the load area, each of which had the nominal power consumption of $100 \mathrm{~kW}$. The load profiles were randomly chosen for a period of 24 hours according to [19]. Most of these assumption are consistent with the microgrid illustrated in [16].

Although microgrids can be also operated in island mode, we assumed in our example that it could still exchange a bidirectional power flow with the external grid supplier, if needed. The V2G programme is activated when the EMS sends the signal to the aggregator that power dispatch from the EVs is required. As long as the signal is not received, the EVs are idle or charged and used as a virtual battery storage device. Finally, we assumed that the CHP is used to balance the power management within the microgrid, if required.

\section{RESUlts AND Discussion}

We assumed that at the beginning of the simulation, 50 EVs are participating to the V2G scheme. Then, we simulated the arrival process of new EVs as a stochastic process where every minute a new EV arrives with probability 5\% until the airport car park is full. The EVs arrive with an initial random $S O C$ greater than $10 \%$, and with a desired $S O C_{\min }$, which 
corresponds to the minimum level of the battery that is strictly required for coming back home from the airport at the end of the journey. If the $S O C_{\min }$ is smaller or equal than the initial $S O C$, then the EV immediately starts participating to the V2G programme, and as illustrated in the algorithm, its $S O C$ will never drop below $S O C_{\min }$. If the $S O C_{\min }$ is greater than the initial $S O C$, then the grid treats the $\mathrm{EV}$ as a normal load, and only when the $S O C$ reaches the level of $S O C_{\min }$ the EV starts participating to the $\mathrm{V} 2 \mathrm{G}$ programme. We assumed that the efficiency parameter of the batteries was different for each $\mathrm{EV}$, and included between $80 \%$ to $90 \%$.

We assumed that the cost of generating power with the CHP was a quadratic function with parameters $\alpha=2, \beta=-3$ and $\gamma=8$. Then, we assumed that the grid would give revenues to the EVs according to another quadratic function with parameters $\alpha_{i}=1, \beta_{i}=-5$ and $\gamma_{i}=8$. With such values, it is always more convenient for the grid to take energy from the EVs than from the CHP (though, obviously, other pricing functions could be chosen to obtain the same result). In addition, we set the iterative update time $\Delta \mathrm{T}$ to 1 minute, which corresponds to the sampling rate of the base load, and the optimisation parameters $\psi$ and $\mu$ were both set to 0.001 .

We now first illustrate the dynamics of the ODC algorithm and analyse the performance of the algorithm from discharging fairness of EVs.Then, we show the benefits for the microgrid after introducing the V2G regulation service by simulation studies.

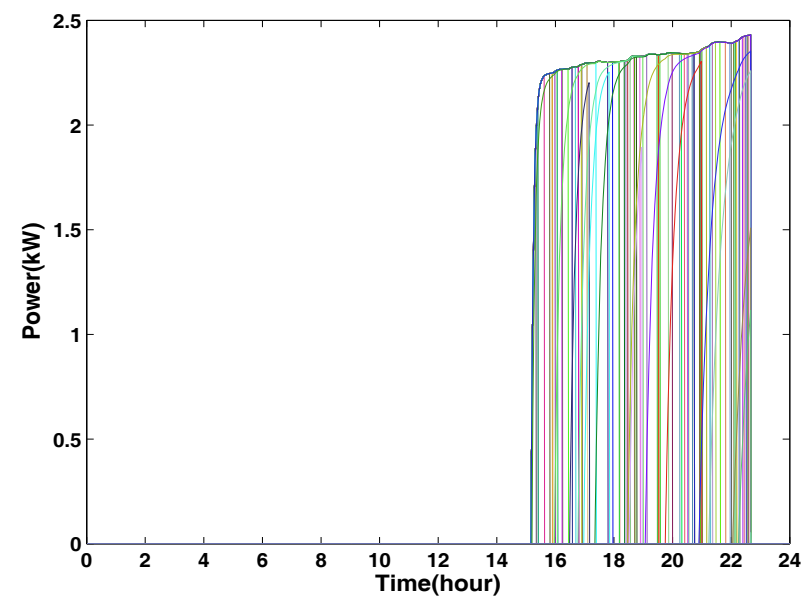

Fig. 4. Discharge rates of the EVs with V2G in operation

Comment: Fig.4 illustrates the discharge rates of all available EVs during the V2G power dispatch. It can be seen that the discharge rates are approximately the same for all the EVs. To better appreciate such a result, we now use the fairness index introduced in [22], which is given by:

$$
F I(t)=\frac{\left(\sum_{i \in \phi(t)} c_{i}(t)\right)^{2}}{|\phi(t)| \cdot \sum_{i \in \phi(t)} c_{i}(t)^{2}}, \forall t \in\{1,2, \ldots, M\}
$$

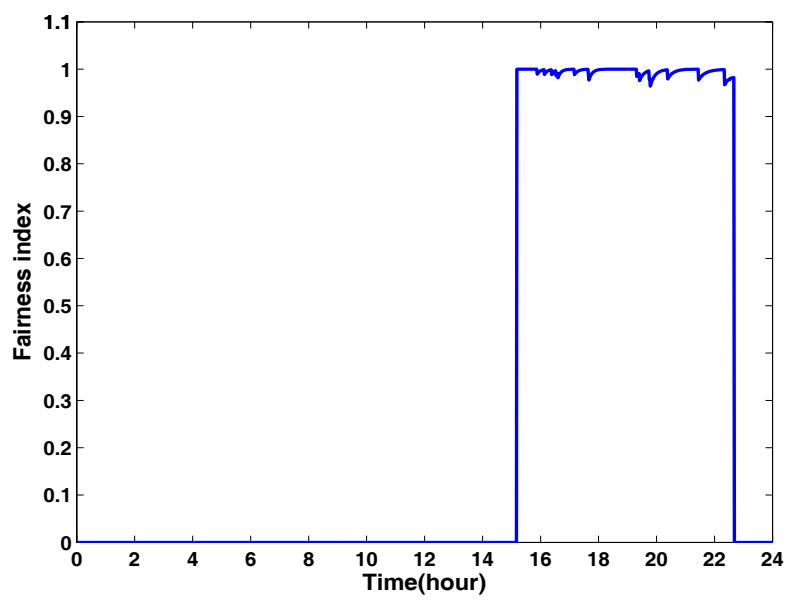

Fig. 5. Fairness index of the ODC algorithm with $\mathrm{V} 2 \mathrm{G}$ in operation

Fig.5 shows that the V2G procedure is fair, apart for some transitory time intervals when a new EV starts participating to the scheme. However, fairness is restored in a very short time. The maximum power that could be generated by each of the DGs is depicted in Fig.6.

The power generated by the DGs is shown in Fig.7. In Fig.7, Totaldemand refers to the power required by the Baseload plus that required by the EVs that are being charged until they reach the required level $S O C_{\min }$. DGmax denotes the maximum power that can be generated by all DGs, DGnoCHP indicates the total maximum power generation by all DGs without considering the CHP, which corresponds to the power generated by renewable sources, and $D G n o C H P+V 2 G$ corresponds to the power generated by renewable sources plus that stored in EVs. In practice, when the total power generated by renewables sources is smaller than the base load, then the EVs with high SOC are discharged. Vice versa, when the total power generated by renewables sources is greater than the base load, then the EVs participating to the $\mathrm{V} 2 \mathrm{G}$ programme are recharged. As a result, Fig.8 shows the evolution of the SOCs of all EVs charging/discharging during the day.

The economical benefits for all stakeholders participating into the V2G scheme are illustrated in Figs.9-14. The economical benefits for the EV owners are demonstrated in Figs.910. Compared to the revenues achieved by the EV owners, it is shown clearly from Figs.11-14 that both EV aggregator and the grid company achieve greater economical advantages by adopting the proposed V2G scheme. According to this analysis, it can be concluded that all stakeholders in the market will be positively encouraged to participating into the scheme for maximising the potential benefits for EVs and renewables.

\section{CONCLUSions}

In this paper we used an optimal distributed consensus algorithm to design a fair $\mathrm{V} 2 \mathrm{G}$ programme. In this programme, a microgrid stores the surplus energy generated by renewable sources into EVs, and take back this energy when needed. The optimality of the V2G strategy is with respect to utility 


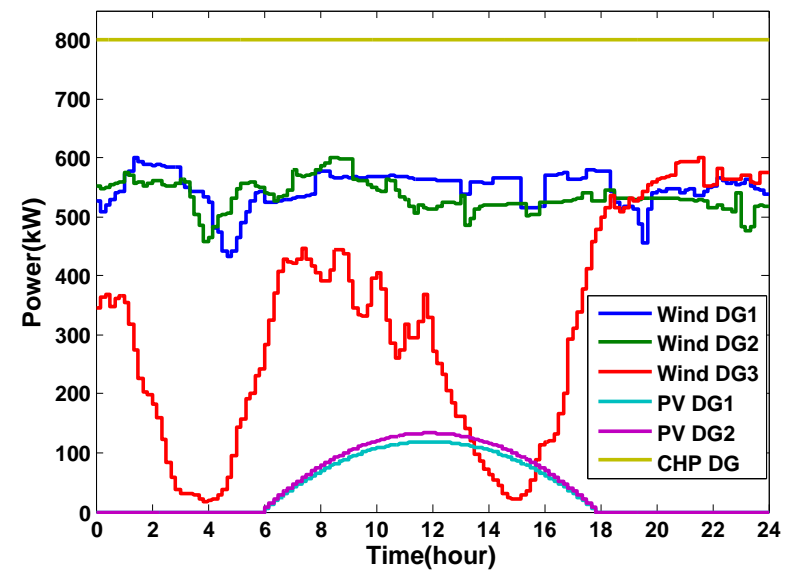

Fig. 6. Power generation profiles of DGs



Fig. 7. Comparison of demand and power generation with $\mathrm{V} 2 \mathrm{G}$ and $\mathrm{EV}$ loads

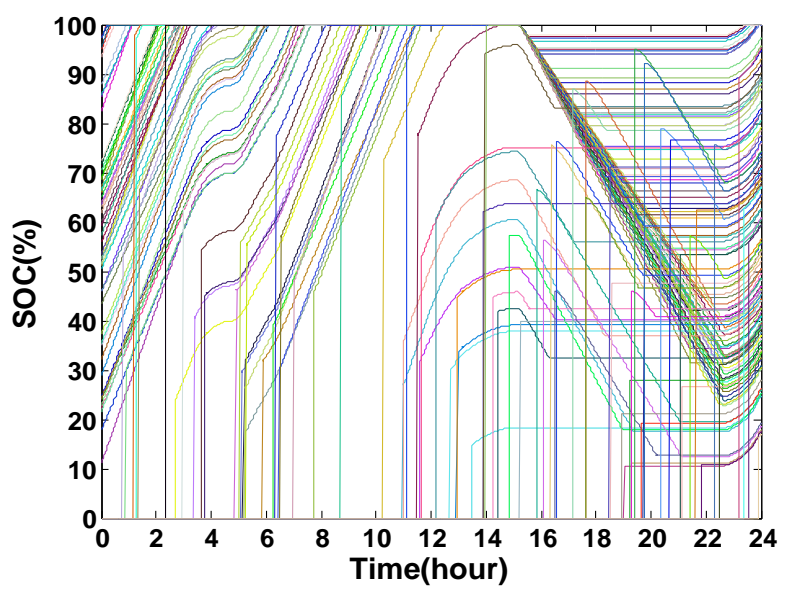

Fig. 8. SOC of the EVs during a day operation



Fig. 9. Accumulative economical benefits for each EV in the scheme

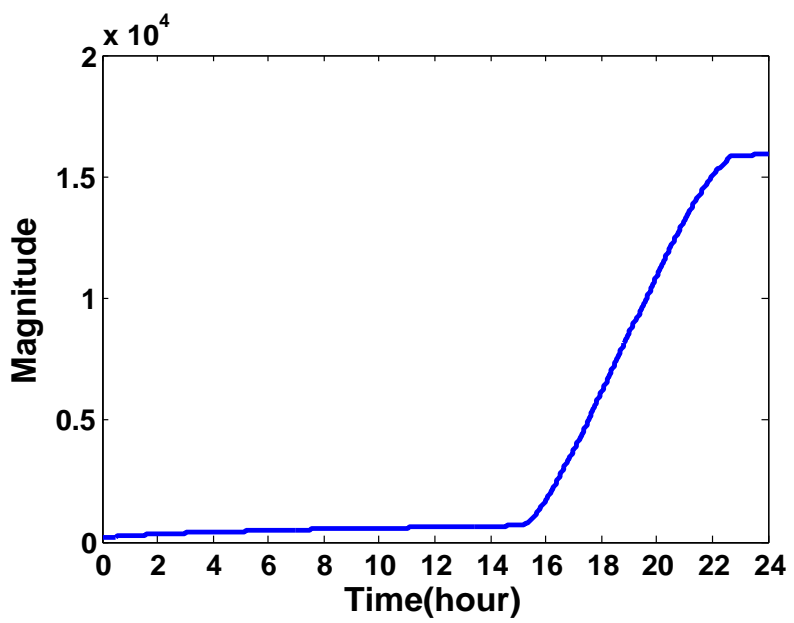

Fig. 10. Accumulative economical benefits for all EVs in the scheme

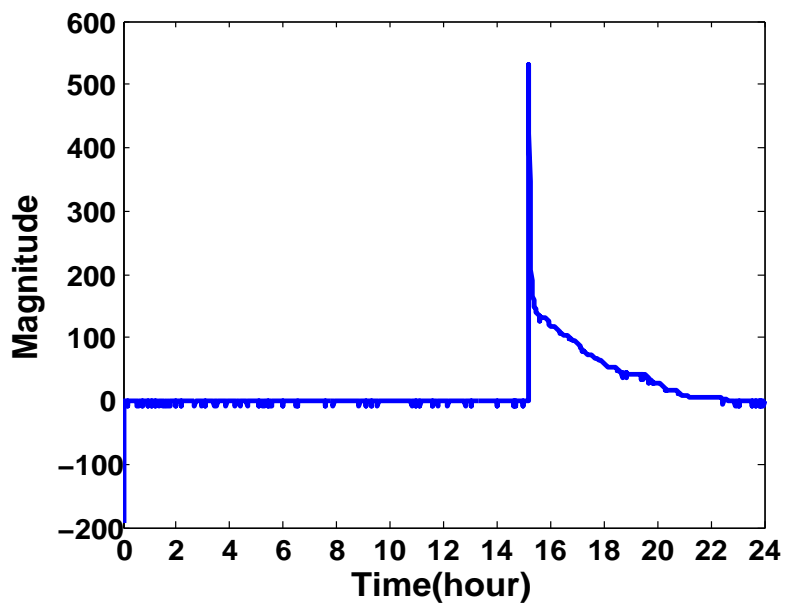

Fig. 11. Economical benefits for the EV aggregator at each time slot 


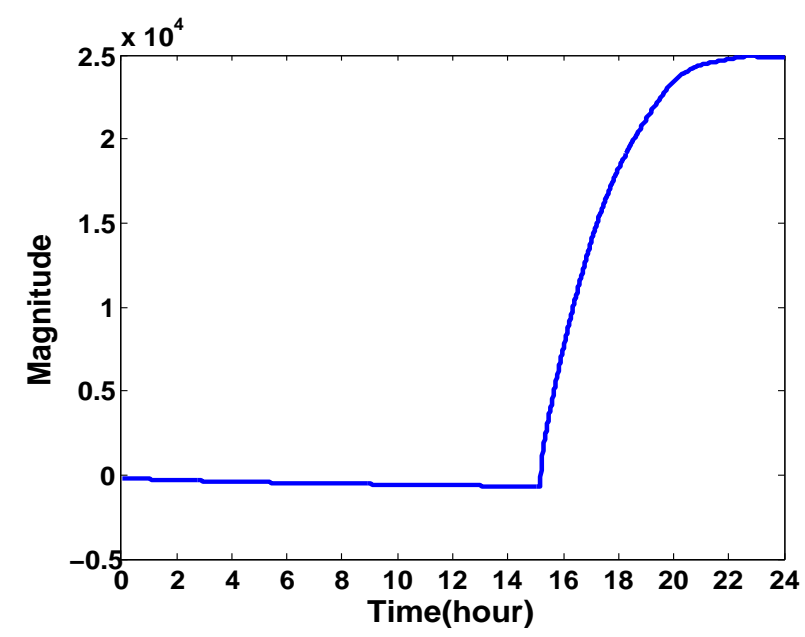

Fig. 12. Accumulative economical benefits for the EV aggregator



Fig. 13. Economical benefits for the grid at each time slot

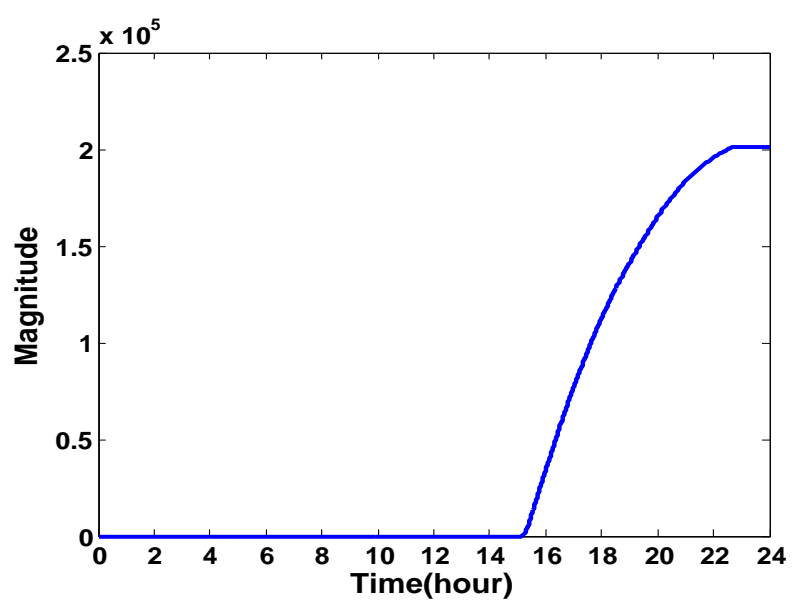

Fig. 14. Accumulative economical benefits for the grid functions that both contain an economic term (the more energy is taken from the EVs, the better for the grid), and a QoS aspect (too much energy should not be taken from the vehicles to avoid getting close to the minimum level of SOC required to safely come back home when the owner takes his/her EV). Then, we formulated the fair power dispatch problem of EVs as a consensus optimisation problem with constraints. The basic algorithm to solve this problem will be discussed in the final paper with detailed proofs on several properties of the algorithm. Then we extended the theoretical algorithm, to include realistic grid constraints, and illustrated the ODC algorithm. Finally, we simulated the ODC algorithm on a dedicated Matlab/OpenDSS testbed, and showed the performance of the algorithm both in terms of fairness and in terms of economic convenience for the grid.

\section{ACKNOWLEDGMENT}

This work was supported by Science Foundation Ireland grant 11/PI/1177.

\section{REFERENCES}

[1] J.A.P. Lopes, F. J. Soares and P. M. R. Almeida, Identifying Management Procedures to Deal with Connection of Electric Vehicles in the Grid, IEEE Bucharest Power Tech Conference, 2009.

[2] S. Deilami, A.S. Masoum, P. S. Moses and M. A. S. Masoum, RealTime Coordination of Plug-In Electric Vehicle Charging in Smart Grids to Minimize Power Losses and Improve Voltage Profile, IEEE Transactions on Smart Grid, Vol. 2, No. 3, 2011.

[3] L. Gan, U. Topcu and S. Low, Optimal Decentralized Protocol for Electric Vehicle Charging, IEEE Control and Decision Conference (CDC), 2011.

[4] K. Mets, T. Verschueren, W. Haerick, C. Develder and F. De Turck, Optimizing smart energy control strategies for plug-in hybrid electric vehicle charging, IEEE/IFIP Network Operation and Management Symposium, pp.293-299, April 2010.

[5] M. Marinelli, F. Sossan, G.T. Costanzo and H.W. Bindner, Testing of a Predictive Control Strategy for Balancing Renewable Sources in a Microgrid, IEEE Transactions on Sustainable Energy, in Press.

[6] P. Meibom, K.B. Hilger, H. Madsen and D. Vinther, Energy Comes Together in Denmark: The Key to a Future Fossil-Free Danish Power System, IEEE Power and Energy Magazine, vol. 11, no. 5, pp. 46-55, 2013.

[7] L. P. Fernández, T.G. San Róman, R. Cossent, C.M. Domingo and P. Frías, Assessment of the Impact of Plug-in Electric Vehicles on Distribution Networks, IEEE Transactions on Power Systems, Vol. 26, No. 1, pp. 206-213, February 2011.

[8] S. Acha, T.C. Green and N. Shah, Effects of Optimised Plug-in Hybrid Vehicle Charging Strategies on Electric Distribution Network Losses, IEEE Transmission and Distribution Conference and Exposition, 2010.

[9] Y. He, B. Venkatesh and L. Guan, Cost Minimization for Charging and Discharging of Electric Vehicles, Interconnections and Communications of Electric Vehicles and Smart Grids, 2011.

[10] S. Stüdli, W. Griggs, E. Crisostomi and R. Shorten, On Optimality Criteria for Reverse Charging of Electric Vehicles, IEEE Transactions on Intelligent Transportation Systems, vol. 15, no. 1, 2014.

[11] OpenDSS Program, Available online through SourceForge.net at URL: http://sourceforge.net/projects/electricdss.

[12] R.H. Lasseter, P. Paigi, Microgrid: a conceptual solution, IEEE 35th Annual Power Electronics Specialists Conference, vol.6, 2004.

[13] What is a Microgrid, Available online through GeneralMicroGrids.com at URL: http://www.generalmicrogrids.com/\#!about-microgrids/c1r3e.

[14] G.E. Asimakopoulou, A.L. Dimeas and N.D. Hatziargyriou, LeaderFollower Strategies for Energy Management of Multi-Microgrids, IEEE Transactions on Smart Grid, vol.4, no.4, pp. 1909-1916, 2013.

[15] P. Venkatesh, R. Gnanadass, and N.P. Padhy, Comparison and application of evolutionary programming techniques to combined economic emission dispatch with line flow constraints, IEEE Transactions on Power Systems, vol.18, no.2, pp. 688-697, 2003. 
[16] E. Crisostomi, M. Liu, M. Raugi, and R. Shorten, Plug-and-Play Distributed Algorithms for Optimised Power Generation in a Microgrid, IEEE Transaction on Smart Grid, vol. 5, no. 4, pp. 2145-2154, 2014.

[17] F. Knorn, Topics in Cooperative Control, Ph.D. dissertation, Hamilton Institute, National University of Ireland Maynooth, 2011.

[18] F. Wirth, M. Liu, R. Shorten, and M. Corless, Consensus on the Feedback, in preparation for submission.

[19] South California Edison, available online at URL: http://www.sce.com/ 005_regul_info/eca/DOMSM11.DLP.

[20] National Sustainable Energy Laboratory (NREL), available online at URL: https://pfs.nrel.gov/main.html.

[21] D. Aloini, E. Crisostomi, M. Raugi and R. Rizzo, Optimal Power Scheduling in a Virtual Power Plant, Innovative Smart Grid Technologies (ISGT) Europe, Manchester, UK, 2011.

[22] J.J. Escudero-Garzas, A. Garcia-Armada, and G. Seco-Granados, Fair Design of Plug-in Electric Vehicles Aggregator for V2G Regulation, IEEE Transactions on Vehicular Technology, vol. 61, no. 8, pp. 34063419,2012 